\title{
Rede Social Google+: Análise de Recursos para a Aprendizagem Colaborativa
}

\author{
George França dos Santos ${ }^{1}$, Mayara Kaynne Fragoso Cabral ${ }^{1,2}$, Paulo César de S. \\ Patrício $^{2}$, Mara Cleide dos Santos ${ }^{3}$, Vanessa A. Corado ${ }^{2}$ \\ ${ }^{1}$ Universidade Federal do Tocantins (UFT) - Programa de Pós-Graduação em \\ Modelagem Computacional / Palmas - TO, Brasil. \\ ${ }^{2}$ Instituto Federal de Educação, Ciência e Tecnologia do Tocantins / Porto Nacional - \\ TO, Brasil. \\ ${ }^{3}$ Instituto Federal de Educação, Ciência e Tecnologia do Tocantins / Palmas - TO, \\ Brasil. \\ george.f@uft.edu.br, \{mayarakf, paulo.patricio, maracleide\}@ifto.edu.br, \\ vanessaa.corado@gmail.com
}

\begin{abstract}
This article aims to identify what features of social network Google+ offer possibilities of use in the school environment, as developers pedagogical use of collaborative learning. For this presents a theoretical study of the concepts involved and an analysis that sought to identify six features considered important for the construction of knowledge: delivering content, activities, evaluation mechanisms, monitoring, privacy, and moderation. The results indicate that the configuration of communities on Google+ is the most suitable option for the practice of educational activities and that the network, except for the possibility of teaching evaluation, displays all features considered important for the construction of knowledge.
\end{abstract}

Resumo. O presente artigo tem como objetivo identificar quais recursos da rede social Google+ oferecem possibilidades de uso no ambiente escolar, como recurso pedagógico fomentador da aprendizagem colaborativa. Para isso apresenta um estudo teórico dos conceitos envolvidos e uma análise que buscou identificar seis recursos considerados importantes para a construção do conhecimento: disponibilização de conteúdo; de atividades; de mecanismos de avaliação; de monitoramento; de privacidade; e de moderação. Os resultados indicam que a configuração de comunidades no Google+é a opção mais adequada para prática de atividades educacionais e ainda que a rede, com exceção da possibilidade de avaliação docente, apresenta todos os recursos considerados importantes para a construção do conhecimento.

\section{Introdução}

Acompanhando o avanço das Tecnologias da Informação e Comunicação (TICs), cada vez mais acessíveis à sociedade civil, o ambiente escolar passa por transformações que visam atender aos aspectos de interação e aprendizado entre todos os atores envolvidos nesse sistema [Mata et al. 2014]. A adição destas novas tecnologias no contexto educacional tem trazido mudanças significativas em todo o processo de aprendizagem [Junior e Pimentel 2013]. E os sites de redes sociais conseguiram grande projeção neste cenário contemporâneo [Ammann 2011]. 


\section{CBIE-LACLO 2015}

Anais dos Workshops do IV Congresso Brasileiro de Informática na Educação (CBIE 2015)

Ferramentas como sites de redes sociais fazem da Web 2.0 um espaço de aprendizagem, sobretudo pela possibilidade de "combinação ou mistura e justaposições desses aplicativos ou ferramentas digitais para formar a base para um ambiente dinâmico e criativo na qual as pessoas possam aprender através de trabalhos colaborativos e coletivos baseados em pesquisas" [Kamel e Wheeler 2007].

Este cenário juntamente com as transformações tecnológicas exige das instituições de educação uma competência inovadora para o ensino, e o abandono das relações verticais herdadas das sociedades industriais e disciplinares do passado.

Com isto este estudo traz como objetivo geral identificar quais recursos disponíveis na rede social Google+ oferecem possibilidades de uso no ambiente escolar, como recurso pedagógico fomentador da aprendizagem colaborativa. Elencaram-se os seguintes objetivos específicos: Identificar como esta rede social pode ser configurada para se adequar a prática de atividades educacionais e verificar quais as capacidades destes recursos para uso na construção do conhecimento.

$\mathrm{Na}$ busca por norteamento para responder aos questionamentos levantados, definiu-se seis recursos considerados importantes na construção do conhecimento em ambientes de redes sociais. São eles: Disponibilização de conteúdo, de atividades, de mecanismos de avaliação, de possibilidades de monitoramento da colaboração, de privacidade, e de moderação.

Este artigo se organiza em cinco seções. Na seção dois é apresentado o procedimento utilizado para a construção da pesquisa e análise das redes sociais; na seção três é realizado o levantamento de materiais bibliográficos e o embasamento conceitual. Na seção quatro é apresentada a análise da rede social e na seção cinco as considerações finais da pesquisa realizada.

\section{Procedimentos para análise}

Para alcançar os objetivos propostos, organizou-se a execução deste estudo em quatro etapas: I) Levantamento de materiais bibliográficos para embasamento conceitual; II) Definição dos critérios de escolha do objeto de estudo; III) Definição dos critérios de análise e IV) Avaliação da rede social.

Assim, para a escolha da rede social a ser analisada como objeto de avaliação levou-se em consideração os seguintes critérios: I) a popularidade do site da rede social na comunidade brasileira; II) a apresentação das características conceituais apresentadas por Body e Ellison (2008), e III) possibilidade de uso em ambiente Web através de computadores.

A Pesquisa Brasileira de Mídia 2015, realizada pelo instituto IBOPE Inteligência foi utilizada como fonte de indicação para as redes sociais consideradas mais populares entre os brasileiros. No ranking o Google + está entre as redes mais populares, e por se enquadrar também nos demais critérios de escolha, foi eleita para ser o objeto de avaliação deste estudo.

Os critérios de análise e avaliação foram definidos com o objetivo de identificar na rede social avaliada recursos com capacidades pedagógicas e fomentadoras da aprendizagem colaborativa. Assim foram definidos seis recursos considerados importantes para a construção do conhecimento, sendo descrito para cada um deles a capacidade esperada: 
I. Conteúdo: capacidade de disponibilizar ao aluno conteúdos como arquivos, vídeos, áudios, textos e etc.

II. Atividades: capacidade de publicação de atividades através da ferramenta de forma que os estudantes possam interagir e colaborar na execução e resposta destas dentro do próprio ambiente.

III. Avaliação: capacidade de registro da avaliação docente identificado a partir do aprendizado ou de algum tipo de produção por parte do estudante adquiridos através dos conteúdos e atividades propostas.

IV. Monitoramento da colaboração: capacidade de acompanhamento da execução das atividades propostas aos alunos, permitindo a identificação de ações colaborativas entre os estudantes.

V. Privacidade: capacidade de sigilo nas publicações das ações realizadas na rede social seja por alunos ou professores.

VI. Moderador: capacidade de moderação por parte do agente pedagógico em relação às postagens dos estudantes, podendo excluir qualquer postagem considerada imprópria.

\section{Redes Sociais e Aprendizagem Colaborativa}

\subsection{Aprendizagem Colaborativa}

A aprendizagem colaborativa pode ser definida como o processo de construção do conhecimento decorrente da participação, do envolvimento e da contribuição ativa dos alunos na aprendizagem uns dos outros [Torres e Amaral 2011].

Este tipo de aprendizagem tem tido várias nomenclaturas: aprendizagem cooperativa, aprendizagem colaborativa, aprendizado coletivo, comunidades de aprendizagem, ensino de pares, aprendizagem entre pares ou aprendizado em equipe. $\mathrm{O}$ que eles têm em comum é que todos eles incorporam o trabalho em grupo [Dooly 2008].

O’Neill, Scott e Conboy (2011) apontaram em sua pesquisa vários estudos que demonstraram a superioridade da aprendizagem colaborativa sobre os modos tradicionais de aprendizagem. Estes estudos indicam que o trabalho em grupo não é apenas uma maneira valiosa de aprendizagem, mas também desenvolve as habilidades para trabalho cooperativo que são essenciais para um local de trabalho moderno.

O objetivo do processo de aprendizagem colaborativa é facilitar a realização de um produto final específico ou meta, através de pessoas que trabalham juntaos em grupos [Dolly 2008]. Mas encontrar a solução de forma colaborativa não é assim tão fácil, como afirma Ballera, Lukandu e Radaw (2013), uma vez que implica em uma adequada comunicação entre os membros no grupo.

Ballera, Lukandu e Radaw (2013) afirmam que neste processo nenhum membro deve se sentir superior ou inferior em relação aos outros; caso contrário um efeito catastrófico no processo de aprendizagem pode ocorrer. A eficiência desta partilha de conhecimentos pode ser alcançada com o arranjo adequado das relações sociais organizacionais [Rózewski et al. 2015].

Outras questões relacionadas com a construção de uma rede de aprendizagem colaborativa incluem os desafios cognitivos, motivacionais e sócio-emocionais além da 
compreensão de como o conflito surge entre os alunos, e que reações e interpretações emocionais estes alunos apresentam [Ayoko et al. 2008; Näykki et al. 2014]. Além disso, pode ainda haver diferenças culturais entre os membros do grupo. De acordo com Popov et al. (2014) quaisquer membro culturalmente diverso dos demais precisa superar um nível adicional de complexidade devido a diferenças relacionadas à cultura.

Grandes são os desafios para se obter uma aprendizagem de forma colaborativa, porém como afirma Różewski et al. (2015) "não há dúvida de que o conceito de colaboração está intimamente relacionado com a aprendizagem".

\subsection{Sites de Redes Sociais (SNS)}

Diferentemente da Web 1.0, que dificultava a troca dinâmica e horizontal de informações, na Web 2.0 existe a bidirecionalidade comunicativa e a não hierarquização do fluxo comunicacional que permite maior interação entre as pessoas [Torres e Amaral 2011]. O autor afirma ainda que a partir do advento da Web 2.0, pode-se entender os meios eletrônicos tanto como instrumentos que impulsionam a formação de redes sociais interativas, horizontais e colaborativas, quanto como instrumentos que alavancam a criação e a disseminação de conhecimentos e aprendizagens.

O amplo termo " rede social " se refere a um grupo de pessoas, muitas vezes, com ênfase nas características interdisciplinares de uma rede, com nodos (pontos de conexão) e vínculos entre eles [Bródka 2013; Fenández 2008). Na internet o termo rede social confere visibilidade aos vínculos da rede de cada usuário, potencializando o grau de comunicação entre os nodos [Fenández 2008].

Um site de rede social (SRS) conforme Body e Ellison (2008) pode ser definido como serviços baseados na web que permitem aos indivíduos: I) construir um perfil público ou semi-público dentro de um sistema limitado; II) articular uma lista de outros usuários com quem esses usuários dividem uma conexão e III) ver e navegar em suas listas de conexões e naquelas feitas por outros no sistema.

Body e Ellison (2008), afirmam ainda que a exibição pública de conexões é um componente crucial do SRSs e que cada site tem seus interesses, tecnologias e perfis de usuários, e que as culturas que surgem em volta dos sites de redes sociais (SRSs) são bem variadas. Enquanto alguns têm compartilhamento de fotos ou vídeos; outros têm tecnologia de mensagens instantâneas; outros são específicos para celulares; outros baseados na web com suporte a interações móveis.

Há muitos exemplos atuais expressivos de sites redes sociais mundialmente acessadas. Segundo Seabra (2010) a cada dia surgem novas redes e outras vão se consolidando à medida que cresce o número de seus usuários e o conceito se dissemina.

\subsection{Relação das redes sociais com a aprendizagem colaborativa}

Aperfeiçoamentos tecnológicos no último quarto de século estimulou um desenvolvimento sem precedentes das ocupações orientadas para o conhecimento, modelos de negócios e paradigmas sociais [Stantchev et al 2015]. Acompanhando este avanço o ambiente escolar passa por transformações com a inserção de tecnologias que visam atender os aspectos de interação e aprendizado entre todos os atores envolvidos nesse sistema [Mata et al. 2014]. 


\section{CBIE-LACLO 2015}

Anais dos Workshops do IV Congresso Brasileiro de Informática na Educação (CBIE 2015)

Lorenzo (2013 p.30) afirma que "embora as redes sociais estejam intrinsecamente voltadas para o entretenimento, percebem-se possibilidades de trabalhos de cunho educacional.".

As redes sociais tornam possível o uso de novas estratégias e ferramentas para apoiar a aprendizagem, oferecendo possibilidades inovadoras para o processo ensinoaprendizagem [Araújo 2010], seu uso na educação serve como impulso na transformação dos paradigmas educacionais, buscando cada vez mais socializar o ensino ao ambiente tecnológico atingindo todas as classes sociais [Thamay e Santos 2013].

Conforme explicado por Ballera, Lukandu e Radaw (2013) muitos educadores acabam se preocupando bastante com a forma como eles devem tratar a mídia social para que não atrapalhem as atividades na sala de aula, e não se atentam que as mídias sociais oferecem recursos que podem construir um ambiente de aprendizagem social de um modo que anteriormente não era possível para resolução de problemas de forma colaborativa.

Muitos são os desafios para o uso das redes sociais no ambiente escolar, mas para enfrentá-los é fundamental que a utilização seja muito bem organizada e planejada, preferencialmente, de forma participativa, entre professores e alunos [Leka e Grinkraut 2014]. Como aponta Lorenzo (2013 p. 105) "Educar é planejar para utilizar bem, com critério, ética e responsabilidade". O uso das redes sociais no processo educativo deve ser feito de maneira bem pensada, para que não seja para os alunos apenas uma distração, gerando mais ruído do que ajudando no processo de ensino aprendizagem [Seabra 2010].

\section{Análise da Rede Social Google+}

Wehunt e Rice (2015) afirmam que a tecnologia de computação em nuvem oferece uma forma excitante e estimulante para aprendizagem transformadora e reforço da comunicação em ambientes de ensino à distância. Afirma ainda que é uma habilidade importante aos educadores aprender como os aplicativos do Google podem efetivamente auxiliá-los a projetar pedagogicamente permitindo a construção social do conhecimento discente.

A rede social mantida pela empresa Google Inc., denominada Google Plus, G+ ou Google+, foi lançada em 2011 com o objetivo de agregar serviços da empresa. Quase todos seus serviços têm conexão ao Google+: Gmail, Drive, Docs, Hangouts e até mesmo as ferramentas de buscas interagem em algum nível com a rede social.

Google+ é uma rede social e mecanismo de recomendação com recursos similares a outras redes como Facebook e Twitter. Como o Facebook, os usuários são capazes de postar e compartilhar com outros usuários conteúdo e atualizações de status. Como no Twitter qualquer pessoa pode acompanhar as mensagens de um determinado usuário sem a necessidade de uma relação direta de amizade para ler os posts escritos por este usuário.

A possibilidade de conversar com diversas pessoas por vídeo, por meio do Hangouts mesmo por um dispositivo móvel a custo zero e com bastante facilidade, é um dos grandes trunfos do Google+. Por meio do Hangouts, o professor pode realizar videoconferência com grupos de até dez estudantes por vez direto da Homepage do Google+ utilizando ferramentas que incluem a ativação de voz e mudo instantâneo. 


\section{CBIE-LACLO 2015}

Anais dos Workshops do IV Congresso Brasileiro de Informática na Educação (CBIE 2015)

Como em outras redes, há o envolvimento de conceitos como "social", "pessoas" e "comunicação", mas com o diferencial de reunir pessoas nos chamados círculos no contexto de muitos dos serviços do Google. Os círculos servem para monitorar o fluxo de informações de diferentes públicos. A pessoa não verá o nome do círculo ao qual ela foi adicionada. A pessoa ou página adicionada poderá ver tudo o que foi compartilhado anteriormente ao seu ingresso com o círculo. Há um limite de até 5.000 perfis e páginas em todos os círculos de um usuário [Google+ 2015].

Erkollar e Oberer (2013) realizaram nos anos de 2011 e 2012 em uma universidade na Turquia uma pesquisa para integrar as principais funcionalidades do Google+ ao ensino superior. Concluíram que o Google+ pode melhorar a colaboração através de círculos, a realização de pesquisas para projetos com alunos, e melhorar a relação do aluno com o instrutor.

Há várias formas de emprego da rede social Google+ que podem ser utilizadas para facilitar os processos de ensino-aprendizagem. A forma mais básica é por meio do próprio perfil pessoal. A rede social, entretanto, apresenta mecanismos mais específicos, que podem funcionar melhor de acordo com o tipo de comunicação que se procura, como o uso de página ou de comunidades.

A configuração de comunidades foi a melhor forma identificada por este estudo para que a rede social possa se adequar à prática de atividades educacionais. Em uma comunidade pode-se adicionar círculos definindo, por exemplo, grupos de alunos ou classes, usuários individuais do Google+ ou endereços de e-mail de qualquer domínio. No Google+ há a possibilidade de condicionar a entrada na comunidade para aqueles membros que possuam e-mail com o domínio da instituição de ensino.

Um recurso interessante é a adição de categorias a comunidade para orientar as discussões e ajudar os estudantes a encontrar os tópicos nos quais estão mais interessados. Um professor pode, por exemplo, ter categorias para cada assunto, ou matéria ministrada.

Assim com o objetivo de verificar quais as possibilidades de uso dos recursos oferecidos pelo Google+ para a construção do conhecimento no ambiente escolar, a rede foi configurada através da criação de uma comunidade com a opção de privacidade fechada. Para a identificação das capacidades dos recursos disponíveis na rede, conforme procedimentos definidos foram avaliados seis recursos:

I. Conteúdo: A rede permite a disponibilização de conteúdo pela publicação de texto, links e compartilhamento de vídeos do Youtube. Porém, identificou-se uma limitação no compartilhamento de arquivos, por meio de upload. Esta ação poderia ser feita pelo Google Drive, ou Google Docs, porém não há em ambos uma integração direta com os membros adicionados à comunidade. Seria necessário usar o recurso de círculos para integrar os serviços.

II. Atividades: Por meio da opção "Texto", pode-se descrever uma atividade ou se criar um debate acerca de qualquer assunto. Não identificamos limite no número de caracteres que poderiam ser utilizados. Ao texto pode se adicionar ainda várias imagens, um link ou um vídeo. Atividades podem ser compartilhadas também por meio da opção "Votação" onde podem ser criadas enquetes limitadas a apenas uma pergunta. 
III. Avaliação: Não foi identificado nenhum recurso que permita a avaliação docente que não a executada manualmente.

IV. Monitoramento da colaboração: Para qualquer postagem é possível visualizar todas as atividades da postagem, incluindo a listagem dos membros que fizeram qualquer tipo de ação relacionada à postagem.

V. Privacidade: As comunidades podem ser restringidas por meio de oito opções, divididas em duas categorias: com restrição ao domínio da organização e sem restrição. As opções de privacidade definem: o tipo de comunidade; quem pode participar; quem pode ver as postagens e os membros; e quem pode encontrar a comunidade através de pesquisas em mecanismos de busca. Pode-se ainda definir o público-alvo, se qualquer pessoa, ou apenas maiores de 18 anos, ou maiores de 21 anos, ou personalizar uma idade mínima. Para uso no ambiente escolar, identificamos que a opção ideal é aquela sem restrição do domínio da escola, onde o tipo de comunidade é particular. Esta opção permite que os estudantes encontrem a comunidade pela pesquisa e solicitem aprovação para participar, mas os moderadores precisam aprovar a participação. Todos os membros podem ver as postagens dos outros membros.

VI. Moderador: Todo o conteúdo é dirigido por seus membros. Mas o proprietário ou moderador de uma comunidade do Google+ pode remover qualquer conteúdo que considerar impróprio ou ofensivo, pode ainda criar categorias para organizar discussões, destacar ótimas postagens, adicionar moderadores para estimular a conversa, convidar membros ou editar a comunidade.

\section{Considerações Finais}

A rede social Google+ oferece várias possibilidades de uso no ambiente escolar, como recursos pedagógicos que podem contribuir para a fomentação da aprendizagem colaborativa, porém será a estratégia pedagógica do professor que vai propiciar que a colaboração aconteça. Com exceção da possibilidade de avaliação docente, todos os demais recursos analisados e definidos como importantes meios para construção da aprendizagem foram identificados na rede.

Através da análise do ambiente da rede foi possível a identificação da criação de comunidades como a melhor opção de configuração para prática de atividades educacionais. $\mathrm{O}$ estudo da capacidade dos recursos disponíveis na rede foi descrito nesta pesquisa e demonstra algumas possibilidades de utilização de cada recurso no ambiente escolar.

Porém para que os recursos oferecidos na rede possam ser utilizados com sucesso no âmbito escolar é necessário que tanto os agentes pedagógicos, como os discentes explorem juntos os potenciais e possibilidades oferecidas pelo Google+. Cabe aos educadores aproveitar a possibilidade aberta, como estratégia pedagógica para acompanhar mais de perto os estudantes e construir, em conjunto com eles, novas possibilidades de uso, e novos processos integradores da formação do conhecimento.

A integração do Google+ com outras ferramentas da empresa Google Inc. garante uma continuidade consistente e um sistema de gerenciamento de conteúdo ideal para o ambiente educacional. O Google+ mostra-se como uma ferramenta alternativa 


\section{CBIE-LACLO 2015}

Anais dos Workshops do IV Congresso Brasileiro de Informática na Educação (CBIE 2015)

para a construção do processo de ensino e aprendizagem, e apropriada para uso profissional e instrucional.

\section{References}

Ammann, M. (2011). Facebook, eu curto: Uma análise mimética das redes sociais digitais. Dissertação de Mestrado em Educação, Faculdade de Educação da Universidade de Brasília, Brasília.

Araújo, D. L. A. (2010). O impacto das redes sociais no processo de ensino e aprendizagem. Anais Eletrônicos do $3^{\circ}$ Simpósio Hipertexto e Tecnologia na Educação. Universidade Federal de Pernambuco - Núcleo de Estudos de Hipertexto e Tecnologias na Educação, Recife-PE, Brasil.

Ayoko, O. B., Callan, V. J., Härtel, C. E. J. (2008). The Influence of Team Emotional Intelligence Climate on Conflict and Team Members' Reactions to Conflict. Small Group Research, Volume 39 Number 2, 121-149, Sage Publications, 10.1177/1046496407304921.

Ballera, M., Lukandu, I. A., Radwan, A. (2013). Collaborative Problem Solving Using Public Social Network Media: Analyzing Student Interaction and its Impact to Learning Process. International Journal of Digital Information and Wireless Communications (IJDIWC) 3(1): 25-42. The Society of Digital Information and Wireless Communications (ISSN: 2225-658X).

Body, M. D., Ellison, N. B. (2008). Social network sites: Definition, history, and scholarship. Journal of Computer Mediated Communication, 13(1), 210-230. International Communication Association.

Bródka, P. (2013). Key User Extraction Based on Telecommunication Data (aka. Key Users in Social Network. How to find them?). arXiv preprint arXiv:1302.1369.

Dooly, M. (2008). Telecollaborative language learning: A guidebook to moderating intercultural collaboration online. Constructing Knowledge Together (21-45). Peter Lang.

Erkollar, A., Oberer, B. J. (2013). Putting Google+ to the Test: Assessing Outcomes for Student Collaboration, Engagement and Success in Higher Education. ProcediaSocial and Behavioral Sciences, 83, 185-189.

Google+ (2015). Encontrar e adicionar pessoas aos círculos. Ajuda do Google+. Disponível em: < https://support.google.com/plus/answer/1047805?hl=pt-BR>. Acesso em 23 jul 2015.

Junior, N. N., \& Pimentel, E. P. (2013). Avaliação da aprendizagem em mídias sociais: como avaliar a colaboração online?. In Anais dos Workshops do Congresso Brasileiro de Informática na Educação (Vol. 2, No. 1).

Kamel B., M. N.,Wheeler, S. (2007). The emerging Web 2.0 social software: an enabling suite of sociable technologies in health and health care education1. Health Information \& Libraries Journal, 24(1), 2-23.

Leka, A. R., Grinkraut, M. L. (2014). A Utilização das Redes Sociais na Educação Superior. Revista Primus Vitam. N 7 - 2º semestre de 2014. ISSN 2236-7799. 


\section{CBIE-LACLO 2015}

Anais dos Workshops do IV Congresso Brasileiro de Informática na Educação (CBIE 2015)

Lorenzo, E. M. (2013) A Utilização das Redes Sociais na Educação: A Importância das Redes Sociais na Educação. 3 ed. São Paulo: Clube de Autores, 126p.

Mata, E. C., Pinheiro, M. F., Jacob Jr, A. F., Costa, J. C., de Santana, Á. L., \& Francês, C. R. (2014). Curso Híbrido usando a Rede Social Facebook no Ensino de Programação de Computadores. In Anais do Simpósio Brasileiro de Informática na Educação (Vol. 25, No. 1, pp. 357-366).

O’Neill, S., Scott, M., Conboy, K. (2011). A Delphi study on collaborative learning in distance education: The faculty perspective. British Journal of Educational Technology, Vol. 42 No 6, 939-949. doi:10.1111/j.1467-8535.2010.01132.x

Pesquisa Brasileira de Mídia (2015). Hábitos de consumo de mídia pela população brasileira. Presidência da República. Secretaria de Comunicação Social. ISBN: 97885-85142-60-5. Brasília.

Popov, V., Noroozi, O., Barrett, J.B., Biemans, H.J.A., Teasley , S.D., Slof B., Mulder, M., (2014). Perceptions and experiences of, and outcomes for, university students in culturally diversified dyads in a computer-supported collaborative learning environment. Computers in Human Behavior, 32, 186-200. ISSN: 0747-5632

Rózewski, P., Jankowskia, J., Bródkab, P., Michalski, R. (2015). Knowledge workers' collaborative learning behavior modeling in an organizational social network. Computers in Human Behavior. Elsevier Ltd. ISSN: 0747-5632

Seabra, C. (2010). Tecnologias na Escola. Telos Empreendimentos Culturais, $1^{\circ}$ edição. ISBN - 978-85-99979-03-7, Porto Alegre.

Stantchev, V., Prieto-González, L., \& Tamm, G. (2015). Cloud computing service for knowledge assessment and studies recommendation in crowdsourcing and collaborative learning environments based on social network analysis. Computers in Human Behavior. ISSN: 0747-5632

Thamay, A. F., Santos, A. (2013). Criação de um curso a distância de Introdução a Eletrônica Digital na Rede Social Educacional REDU. In Anais do Simpósio Brasileiro de Informática na Educação (Vol. 24, No. 1).

Torres, T. Z., Amaral, S. F. (2011). Aprendizagem Colaborativa e Web 2.0:Proposta de Modelo de Organização de Conteúdos Interativos. ETD - Educação Temática Digital , 12, 49-72.

Wehunt, M. D., \& Rice, L. (2015). Finding collaborative online tools daunting? Essential tips for navigating the evolving Google+ landscape. The Researcher, 27(1), 31-33. 